\title{
El problema de la gestión hospitalaria de pacientes ectópicos
}

\author{
Fernando Fernández Lamelo \\ Director de Procesos con Ingreso \\ EOXI Lugo, Cervo y Monforte de Lemos
}

El proceso tradicional de hospitalización de pacientes en unidades de Enfermería asignados a determinadas especialidades médicas ha influido en la organización de la asistencia sanitaria, facilitando el trabajo en equipo, la adquisición de conocimientos específicos a nivel médico y los cuidados de enfermería. Además, permite a su vez gestionar de manera adecuada los programas de atención en dispositivos alternativos a la hospitalización convencional, disminuyendo el número de estancias inapropiadas.

La existencia de pacientes periféricos o ectópicos, esto es, aquellos ingresados a cargo de un servicio en camas asignadas a otro, se asocia a efectos asistenciales negativos. Si bien no existe acuerdo en cuanto sus consecuencias en las complicaciones o en la mortalidad, los estudios realizados coinciden en que la asistencia de pacientes ectópicos se asocia a un aumento de las estancias y de los reingresos, como muestra el estudio de Montero et al publicado en este número de Galicia Clínica ${ }^{1-3}$.

Es evidente que el paciente ingresado ectópico distorsiona la normal organización de la Unidad de Enfermería receptora del mismo, que tendrá que recordar o aprender planes de cuidados no habituales, nuevas pautas terapéuticas de otros facultativos y que el servicio médico responsable deberá asumir una sobrecarga al atender más pacientes y en unidades de Enfermería no habituales para los mismos. Esta circunstancia se produce con más frecuencia en pacientes de especialidades médicas, especialmente Medicina Interna, debido a que la situación que los genera se asocia habitualmente al concepto de ingreso urgente ${ }^{1}$.

La presión a la que está sometido el servicio de urgencias tiene una repercusión directa en la gestión de camas debido a los ingresos que de él derivan. Está sometido a una demanda creciente que favorece en ocasiones largas esperas que generan malestar e incertidumbre en pacientes y familias. Por otra parte, a la necesidad de atender la dinámica inherente al dispositivo asistencial, se suma la dificultad de proporcionar adecuados cuidados médicos y de enfermería a los pacientes en espera de decisión clínica o ingreso. En este sentido, existen diferencias entre los tiempos de espera reales y los percibidos, de tal manera que los percibidos aumentan al estar en camilla respecto a una cama, a la intensidad del dolor o a la autopercepción de una mayor gravedad. También pueden variar en función del horario (mayor por las mañanas) y del tiempo en ser atendido por un médico por primera vez. Por ello, una correcta gestión hospitalaria debe tener en cuenta la responsabilidad del personal sanitario y los derechos del paciente. Es más fácil hacer efectivo el respeto a la intimidad y a la confidencialidad, así como mejorar la sensación percibida por un paciente que debe ser ingresado, en una cama de una unidad de hospitalización ${ }^{4}$.

Existen dos modelos en la gestión de camas hospitalarias: el modelo tradicional de asignación a un servicio determinado, que es más rígido y puede crear conflicto ante la necesidad urgente de asignación de una cama, y el flexible con mayor tendencia a generar pacientes ectópicos 5 . Por tanto, la gestión de camas debe provenir de un conocimiento profundo de los circuitos de alta hospitalaria y de ingreso desde urgencias, ya que ambos están lógicamente interconectados. Así, es preciso tener en cuenta una serie de aspectos:

- Conocer número de camas disponibles totales en el centro y asignados a cada especialidad, contemplando las esperas programadas y urgentes. Es obligado disponer de datos históricos y dinámicos actualizados que tengan en cuenta diversas variables: número de urgencias atendidas, número de urgencias ingresadas, estancias, índice de ocupación, índice de rotación y estancia media.

- Conocer en tiempo real la situación de las camas de hospitalización. Este aspecto hace imprescindible la implicación, colaboración y apoyo de todo el personal facultativo durante el proceso completo de hospitalización. Por una parte, es necesario mejorar la eficiencia en la gestión de los recursos asistenciales, como disminuir las 
estancias inadecuadas en espera de pruebas que se pueden realizar de manera ambulatoria, agilizar la detección precoz de problemas sociales, evaluar adecuadamente la necesidad de transporte sanitario, comunicar las previsiones de altas hospitalaria con tiempo de antelación, etc. Por otra, es obligado disponer de un sistema informático ágil que permita que la información fluya en tiempo real para la toma de decisiones adecuadas.

- Conocer la situación clínica del paciente al que se va o asignar la cama.

Todo este proceso debe estar controlado por una estructura hospitalaria estable en el tiempo y responsable último de la asignación del recurso cama, el servicio de Admisión. En este sentido hay es fundamental la labor de personal de enfermería asignado este servicio, con un papel fundamental en la coordinación con las supervisoras de las distintas unidades de Enfermería y con el servicio de Urgencias, dando prioridad clínica al ingreso y asignándole la cama más apropiada.

La oferta del recurso cama no depende sólo de la cantidad, sino del uso que se haga de ella. Tanto gestores como profesionales sanitarios debemos implicarnos en su gestión, de otra manera será imposible modificar el círculo vicioso que se genera cuando el retraso en el proceso de alta de hospitalización de un paciente repercute directamente en la demora de un ingreso desde el servicio de Urgencias y favorece que éste se realice en una unidad diferente de la apropiada. Es preciso trabajar conjuntamente en el rediseño del proceso para mejorarlo y darle más flexibilidad, sobre todo en los períodos del año en los que sabemos sistemáticamente que se produce un aumento de los ingresos en los servicios médicos (ej. épocas de epidemia gripal). En este sentido, debemos disponer de planes perfectamente trazados y organizados que determinen la necesidad de aumento de recursos tanto materiales como profesionales para no generar ingresos ectópicos, con control exhaustivo de aislamientos e intensificación de medidas diseñadas para la atención en dispositivos alternativos a la hospitalización convencional (Hospitales de Día, Hospitalización a Domicilio).

Por otra parte, es especialmente importante trabajar en una definición clara de todos los flujos ambulatorios de pacientes con programas consensuados entre facultativos de Atención Primaria y las distintas especialidades hospitalarias para todas aquellas patologías que por su potencial gravedad requieran una atención prioritaria y en programas de asistencia compartida dirigida a pacientes crónicos complejos. Todas estas medidas redundarán sin duda no sólo en una mejora de la atención dispensada sino también en disminuir presión de los servicios de Urgencias y en la necesidad de estancias inapropiadas en unidades de hospitalización.

En definitiva, la gestión de las camas hospitalarias y por tanto del problema de los pacientes ectópicos es compleja y tiene múltiples caras e implicaciones. Como la mayoría de los problemas que subyacen en el día a día hospitalario debe contar con la colaboración transversal de gestores y profesionales sanitarios. También en este aspecto debemos formar equipos multidisciplinares centrados en el paciente.

\section{Referencias}

1. Cabrera Torres E, García Iglesias MA, Santos Jiménez MT, González Hierro M, Diego Domínguez ML. Los ingresos ectópicos y su relación con la aparición de complicaciones y estancias prolongadas. Gac Sanit. 2019 Jan - Feb;33(1):32-37

2. Perimal-Lewis L, Li JY, Hakendorf PH, Ben-Tovim DI, Qin S, Thompson CH. Relationship between in-hospital location and outcomes of care in patients of a large general medical service. Intern Med J. 2013 Jun;43(6):712-6

3. Montero Ruiz E, Pérez Sánchez L, Barbero Allende JM, Agudo Alonso R, Rebollar Merino A, López Álvarez J. Resultados asistenciales y económicos en los pacientes periféricos de medicina interna. Galicia Clin

4. Asenjo MA. Las claves de la gestión hospitalaria. Barcelona. Ediciones Gestión 2000,1999

5. Cosialls D. Admisiones hospitalarias. Barcelona. Mosby/Doyma libros SA, 1995 